\title{
Abkürzungen der Verlagsorte
}

\begin{tabular}{|c|c|c|c|}
\hline Augsb. & Augsburg & Leningr. & Leningrad \\
\hline BIn & Berlin & Lond. & London \\
\hline Bln-G.-H. & Berlin-Göttingen-Heidelberg & Lpz. & Leipzig \\
\hline Bambg. & Bamberg & Madr. & Madrid \\
\hline Brsehw. & Braunschweig & Magdeb. & Magdeburg \\
\hline Bresl. & Breslau & Mannh. & Mannheim \\
\hline Darmst. & Darmstadt & Marb. & Marburg \\
\hline Dortm. & Dortmund & Mosk. & Moskau \\
\hline Dsdn & Dresden & Müneh. & München \\
\hline Dsdn-Lpz. & Dresden-Leipzig & Nürnb. & Nürnberg \\
\hline Düsseld. & Düsseldorf & Oldenb. & Oldenburg \\
\hline Frkf./M. & Frankfurt/Main & Pdborn & Paderborn \\
\hline Frkf./0. & Frankfurt/Oder & Rgsb. & Regensburg \\
\hline Gött. & Göttingen & Rost. & Rostock \\
\hline Grelfow. & Greifswald & Salab. & Salzburg \\
\hline Hambg & Hamburg & Stockh. & Stockholm \\
\hline Hannov. & Hannover & Stuttg. & Stuttgart \\
\hline Heldelb. & Heidelberg & Tüb. & Tübingen \\
\hline Karlsr. & $\begin{array}{l}\text { Karlsruhe } \\
\text { Königsberg/Pr. }\end{array}$ & Welnh./Bergstr. & Weinheim/Bergstr. \\
\hline Konst. & Konstanz & Wolfenb. & Wolfenbüttel \\
\hline Langens. & Langensalza & Würzb. & Würzburg \\
\hline
\end{tabular}

\section{Häufig gebrauchte Textkürzungen}

\begin{tabular}{|c|c|c|c|}
\hline $\begin{array}{l}\text { Akad. } \\
\text { akt. } \\
\text { algebr. } \\
\text { allg. } \\
\text { Alm. } \\
\text { Amer. } \\
\text { amtl. } \\
\text { analyt. } \\
\text { Anat. } \\
\text { anatom. } \\
\text { Angest. } \\
\text { angew. } \\
\text { Anm. } \\
\text { anorg. } \\
\text { Anst. } \\
\text { ao. Prof. } \\
\text { apl. Prof. } \\
\text { Apoth. } \\
\text { Appar. } \\
\text { Apr. } \\
\text { Arb. }\end{array}$ & $\begin{array}{l}\text { Abbildung } \\
\text { Abhandlung(en) } \\
\text { Abkömmling } \\
\text { Abschnitt } \\
\text { absolut } \\
\text { Abteilung } \\
\text { Abteilungsleiter } \\
\text { Abteilungs vorstand } \\
\text { auBer Dienst } \\
\text { Algemeine Elektrizitätsgesell- } \\
\text { schaft } \\
\text { Aktiengesellschaft } \\
\text { Aktiengesellschaft f. Anilin- } \\
\text { fabrikation } \\
\text { Akademie } \\
\text { aktiv } \\
\text { algebraisch } \\
\text { allgemein } \\
\text { Almanach } \\
\text { Amerika (amerikanisch) } \\
\text { amtlich } \\
\text { analytisch } \\
\text { Anatomie } \\
\text { anatomisch } \\
\text { Angestellter } \\
\text { angewandt } \\
\text { Anmerkung } \\
\text { anorganisch } \\
\text { Anstalt } \\
\text { auBerordentl. Professor } \\
\text { auBerplanmäB. Professor } \\
\text { Apotheke, Apotheker } \\
\text { Apparat } \\
\text { April } \\
\text { Arbeit, Arbeiter }\end{array}$ & $\begin{array}{l}\text { Arch. } \\
\text { Ass. } \\
\text { Astrol. } \\
\text { Astron. } \\
\text { Astrophys. } \\
\text { Atmosph. } \\
\text { Atomgew. } \\
\text { Aufg. } \\
\text { Aufl. } \\
\text { Aug. } \\
\text { Ausg. } \\
\text { ausl. } \\
\text { auBerd. } \\
\text { Autobiogr. } \\
\text { BA. } \\
\text { b. } \\
\text { Ballist. } \\
\text { balt. } \\
\text { bayer. } \\
\text { Bd } \\
\text { Bde } \\
\text { Bearb. } \\
\text { Beauftr. } \\
\text { Beeld. } \\
\text { Beih. } \\
\text { Beil. } \\
\text { Beigp. } \\
\text { Beltr. } \\
\text { belieb. } \\
\text { Bem. } \\
\text { Beob. } \\
\text { Ber. } \\
\text { berat. } \\
\text { Berïeks. }\end{array}$ & $\begin{array}{l}\text { Archiv } \\
\text { Assistent } \\
\text { Astrologie (astrologisch) } \\
\text { Astronomie (astronomisch) } \\
\text { Astrophysik } \\
\text { Atmosphäre } \\
\text { Atomgewicht } \\
\text { Aufgabe } \\
\text { Auflage } \\
\text { August } \\
\text { Ausgabe } \\
\text { ausländisch } \\
\text { auBerdem } \\
\text { Autobiographie } \\
\text { Bergakademie } \\
\text { bei, beim } \\
\text { Ballistik } \\
\text { baltisch } \\
\text { bayerisch } \\
\text { Band } \\
\text { Bände } \\
\text { Bearbeiter } \\
\text { Beauftragter } \\
\text { Beeidigter } \\
\text { Beiheft } \\
\text { Beilage } \\
\text { Beispiel } \\
\text { Beitrag } \\
\text { beliebig } \\
\text { Bemerkung } \\
\text { Beobachtung } \\
\text { Bericht } \\
\text { beratender } \\
\text { Berücksichtigung }\end{array}$ \\
\hline
\end{tabular}




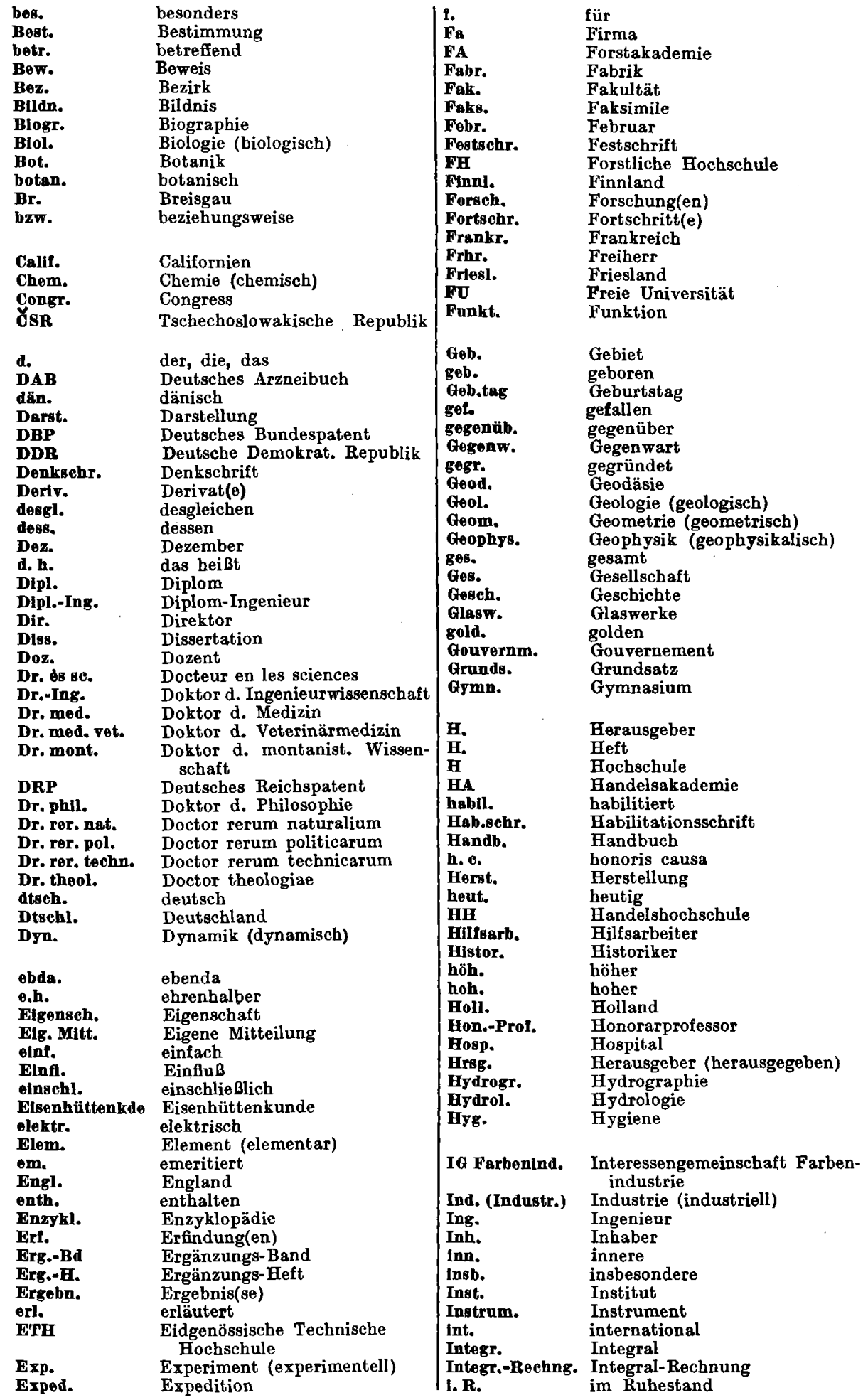




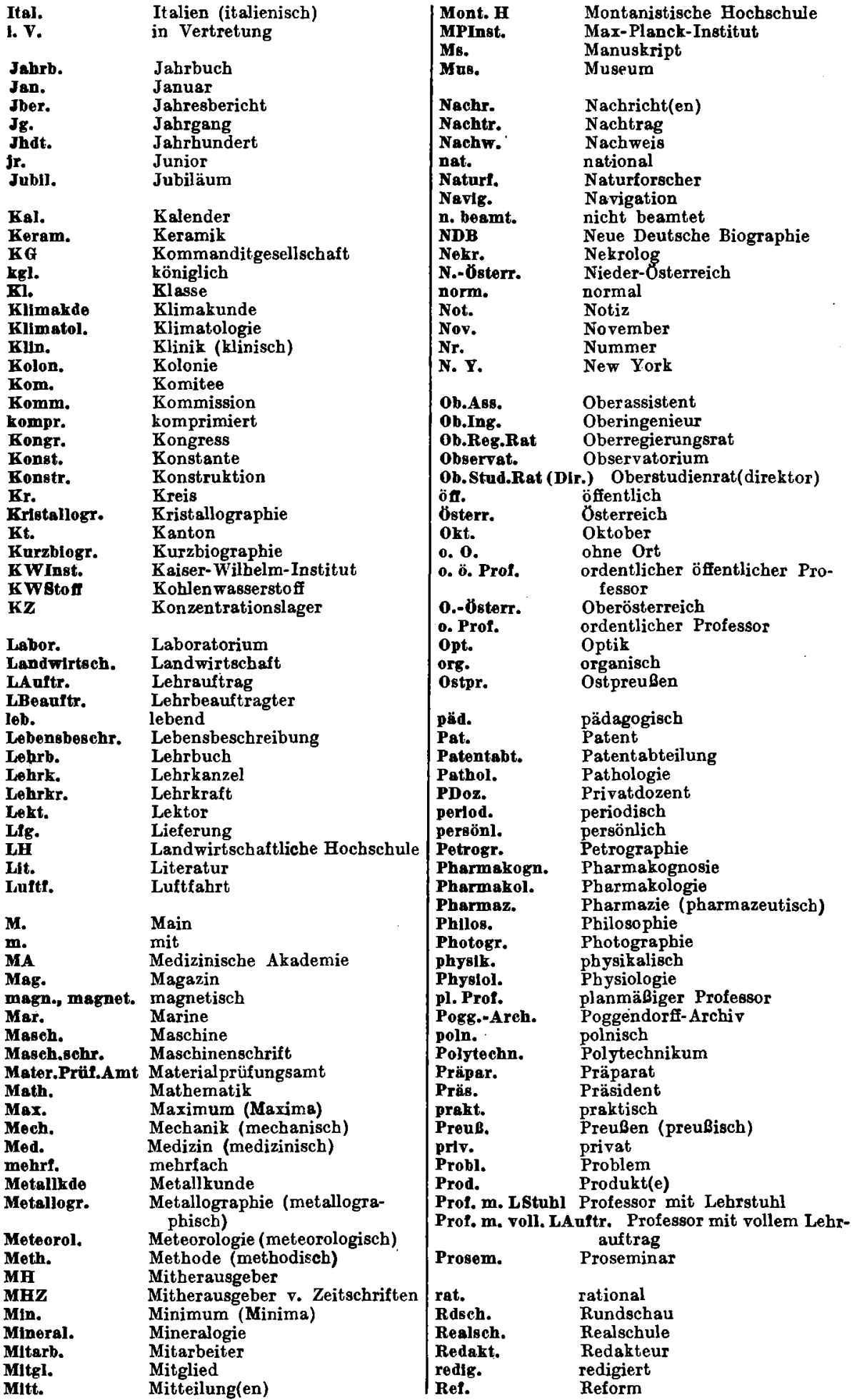




\begin{tabular}{|c|c|c|c|}
\hline $\begin{array}{l}\text { S. } \\
\text { s. } \\
\text { Sa. } \\
\text { Schles. } \\
\text { Sehriftl. } \\
\text { Sehriftst. } \\
\text { Schr.verz. } \\
\text { gchwelz. } \\
\text { sd } \\
\text { Seew. } \\
\text { seitd. } \\
\text { Sekret. } \\
\text { Sem. } \\
\text { sen. } \\
\text { Sept. } \\
\text { Ser. } \\
\text { Slg. } \\
\text { sogen. } \\
\text { soz. } \\
\text { Spektr. } \\
\text { spez. } \\
\text { St. } \\
\text { steatl. } \\
\text { Standp. } \\
\text { Stat. } \\
\text { Stellv. } \\
\text { Sternw. } \\
\text { Strukt. } \\
\text { Stud. } \\
\text { Stud.-Rat } \\
\text { Subot. } \\
\text { Suppl. } \\
\text { Syst. } \\
\text { Synth. }\end{array}$ & 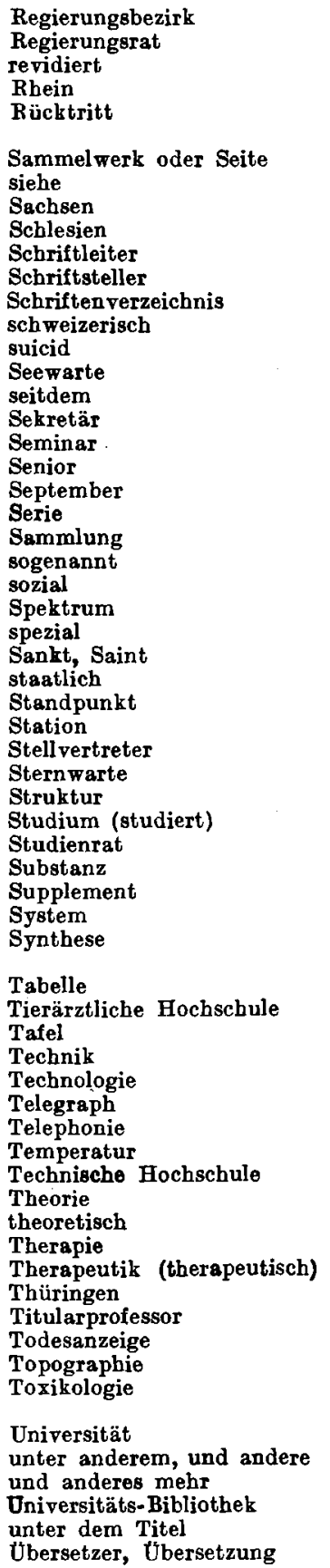 & $\begin{array}{l}\text { univ. } \\
\text { unt. } \\
\text { Unterr. } \\
\text { Unters. } \\
\text { Unters.Abt. } \\
\text { USA } \\
\text { USsR } \\
\text { v. } \\
\text { VDE } \\
\text { VDI } \\
\text { Ver. } \\
\text { Verb., Verbb. } \\
\text { Verf. } \\
\text { vergl. } \\
\text { Verh. } \\
\text { Vermess. } \\
\text { Vermessungswes } \\
\text { Veröf. } \\
\text { Vers. } \\
\text { Vers.Abt. } \\
\text { Vers.Anst. } \\
\text { verseh. } \\
\text { Versich. Ges. } \\
\text { Versich.Math. } \\
\text { Verz. } \\
\text { vielf. } \\
\text { vlol. } \\
\text { Vlzepräs. } \\
\text { Vjschr. } \\
\text { vol. } \\
\text { vorh. } \\
\text { Vork. } \\
\text { vorm. } \\
\text { Vors. } \\
\text { Vorschr. } \\
\text { Vorst. } \\
\text { Vortr. } \\
\text { w. } \\
\text { Wbl. } \\
\text { Wes. } \\
\text { Westf. } \\
\text { Wetterw. } \\
\text { wirkl. } \\
\text { Wirtseh. } \\
\text { Wiss. } \\
\text { WBchr. } \\
\text { Württ. } \\
\text { Z. } \\
\text { z. } \\
\text { zahlr. } \\
\text { Zentr. } \\
\text { zerlegb. } \\
\text { Zivlling. } \\
\text { Ztg. } \\
\text { zugl. } \\
\text { zus. } \\
\text { zw. }\end{array}$ & $\begin{array}{l}\text { Ungarn } \\
\text { universell } \\
\text { unter } \\
\text { Unterricht } \\
\text { Untersuchung } \\
\text { Untersuchungsabteilung } \\
\text { United States of America } \\
\text { Union d. soz. Sowjet-Republiken } \\
\text { von, rom } \\
\text { Verband Deutscher Elektro- } \\
\text { techniker } \\
\text { Verband Deutscher Ingenieure } \\
\text { Verein } \\
\text { Verbindung, Verbindungen } \\
\text { Verfahren } \\
\text { vergleichende } \\
\text { Verhandlung(en) } \\
\text { Vermessung } \\
\text { Vermessungswesen } \\
\text { Veröfentlichung } \\
\text { Versuch(e) } \\
\text { Versuchsabteilung } \\
\text { Versuchsanstalt } \\
\text { verschieden } \\
\text { Versicherungsgesellschaft } \\
\text { Versicherungsmathematik } \\
\text { Verzeichnis } \\
\text { vielfach } \\
\text { violett } \\
\text { Vizepräsident } \\
\text { Vierteljahrsschrift } \\
\text { volume } \\
\text { vorhanden } \\
\text { Vorkommen } \\
\text { vormals } \\
\text { Vorsitzender } \\
\text { Vorschrift } \\
\text { Vorstand } \\
\text { Vortrag } \\
\text { Werke } \\
\text { Wochenblatt } \\
\text { Wesen } \\
\text { Westfalen } \\
\text { Wetterwarte } \\
\text { wirklich } \\
\text { Wirtschaft (wirtschaftlich) } \\
\text { Wissenschaft (wissenschaftlich) } \\
\text { Wochenschrift } \\
\text { Württemberg } \\
\text { Zeitschrift } \\
\text { zum, zur } \\
\text { zahlreich } \\
\text { Zentrum } \\
\text { zerlegbar } \\
\text { Zivilingenieur } \\
\text { Zeitung } \\
\text { zugleich } \\
\text { zusammen } \\
\text { zwischen }\end{array}$ \\
\hline
\end{tabular}

